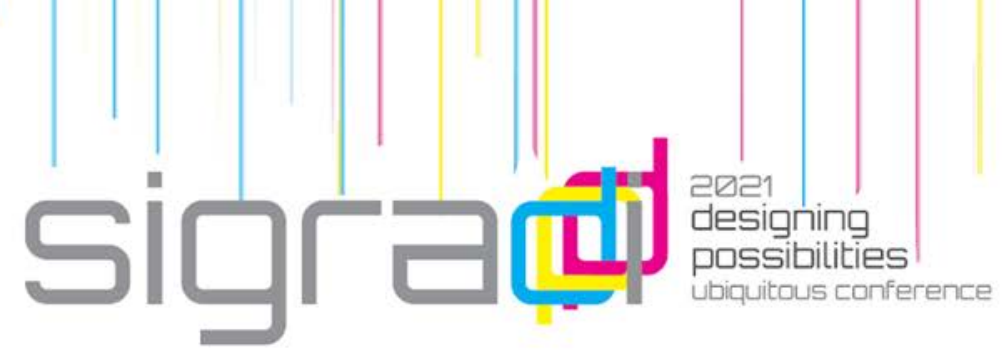

\title{
Robotic Weaving Manufacturing of Optimized Glass Fibres Panels
}

\author{
Tomas Vivanco ${ }^{1}$, Philip Yuan ${ }^{2}$ \\ ${ }^{1}$ Pontifical Catholic University of Chile, Chile \\ Tongji University, China \\ tvivancol@uc.cl \\ 2 Tongji University, China \\ philipyuan007@tongii.edu.cn
}

\begin{abstract}
This article presents the development of robotically fabricated and topological optimized fibreglass weaved panels. The process was led under the material intelligence workflow composed of the digital simulation of mechanical behaviour of the component, programming and optimization of the toolpath, and digital manufacturing with a common CNC machine. Through this process, the panels are optimized to minimize the use of material, decreasing the production time, to achieve its maximum mechanical and functional performance within its own design space.
\end{abstract}

Keywords: Robotic manufacturing, optimization, fiberglass.

\section{Introduction}

Composite materials offer a wide range of uses in construction, the flexibility to make different shapes, their mechanical properties and extended useful life make them high-performance materials with comparative advantages over other conventional materials -like aluminium and iron. The application of reinforced plastics with carbon or glass fibers have been very successful in the Aerospatiale, and the past years, in the AEC industries, offering the advantage of having the same -and in some cases better - mechanical properties of components for a fraction of its weight (Burry et al., 2020) compared with conventional materials.

These advances had open new design possibilities for architectural components, opening the development of computational design and robotic fabrication processes to manufacture efficient material systems. Buga (Damborsio, et al., 2020) and Elytra (Dörstelmann, 2017) Pavilions, both developed by researchers from the University of Stuttgart, presents a clear path for the integration of design, simulation, and advanced robotic technologies and processes that can lead to non-standard three-dimensional structures, almost 


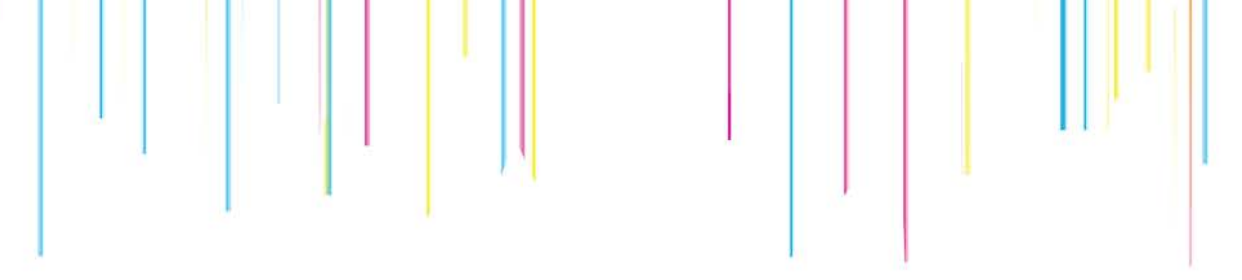

impossible to manufacture with common materials and processes because of its precision, energetic and structural efficiency (Burry, et al., 2020).

From a computational design perspective, each fiber can be understood as a vector (Knippers, 2014) where the internal mechanical forces can be homogeneously distributed through the component (Hensel, 2008). Generative Design for topological mechanical optimization in two dimensions of vector forces has been successfully deployed in the Bionic project developed by The Living in 2016, where the goal was to decrease in 30\% the weight of an Airbus A320 internal partition. Each of the internal structural bars was optimized and being replaced with a set of small bars with different thicknesses and strength dependents on the mechanical stresses and loads. One of the problems faced was the standardization of the production of each bar of the partitions, making the process complex for a very straightforward problem.

One of the negative aspects of petrol-based composites materials is their harmful impact on the environment for their manufacturing and their low second life extension possibilities after their original use, reducing its circularity. Nevertheless, advanced in the development of reinforced fibers polymers (NFRP), based on lignocellulosic fibers combined with basic digital manufactured formworks as a complementary tool for manual processes like weaving (Dahy, 2019), have been used successfully in the design and fabrication of high performance and -low tech material systems components (Jaafar, 2019). Making the manufacturing process more accessible in terms of equipment, diverse in terms of material and less harmful for the environment.

Both manufacturing technics, manual and advanced robotics, offers a wide spectrum of possibilities for composite materials. The integration of both processes might allow the serial production of personalized prefabricated structural composite components (Hensel, 2012) to replace, for example, partitions or structural walls commonly used in the construction industry with high structural performance, reducing to the minimum its weight, and with less use of energy (Vivanco, 2020).

This research proposes a straightforward computational design and robotic manufacturing workflow to produce energy-efficient and high mechanical performance fiber panels (Dambrosio, et al., 2020) with common digital fabrication equipment like a three-axis milling machine. 


\section{Methods and processes}

As an initial state to develop this research, studies, and selection of materials and their processes to work with were defined. Further steps were held under an iterative and analytical process between digital and physical prototypes.

1. Composite mixture definition: Roving fiberglass filament, polymeric resin recipe composed by initiators, promoters, accelerators and retarders, and support matrix.

2. CNC Weaving tool: Design and development of a custom and universal weaving tool that can adjust to (almost) any CNC Milling Machine that can keep a constant tension over the fiber and wet the fiber with the polymeric resin in a liquid state, in a pultrusion process.

3. Computational design for mechanical simulation and topological optimization based on machine-learning of structural fibers panels.

4. Fabricate physical prototypes with a three-axis CNC milling machine adapted with the custom weaving tool.

\subsection{Composite mixture}

There are general recommendations as referenced values for the polymer-fiber rigid matrix (Ashland, 2006), but as the curing process is done with ambient temperature, it is necessary to develop empiric tests (Table 1), to ensure stable, comparable, and desired results, due to the different environmental agents that might affect the mixture composed by initiators, promoters, accelerators, and retarders. Also, to include manufacturing variables like the machine movement speed, quantity and density of the vectors, both with a direct incidence over the time variable. Because of this, the initial mixture had an increment on the cobalt naphthenate (CoNAP), calculated to have a stable liquid mixture for approximately 20 minutes. 

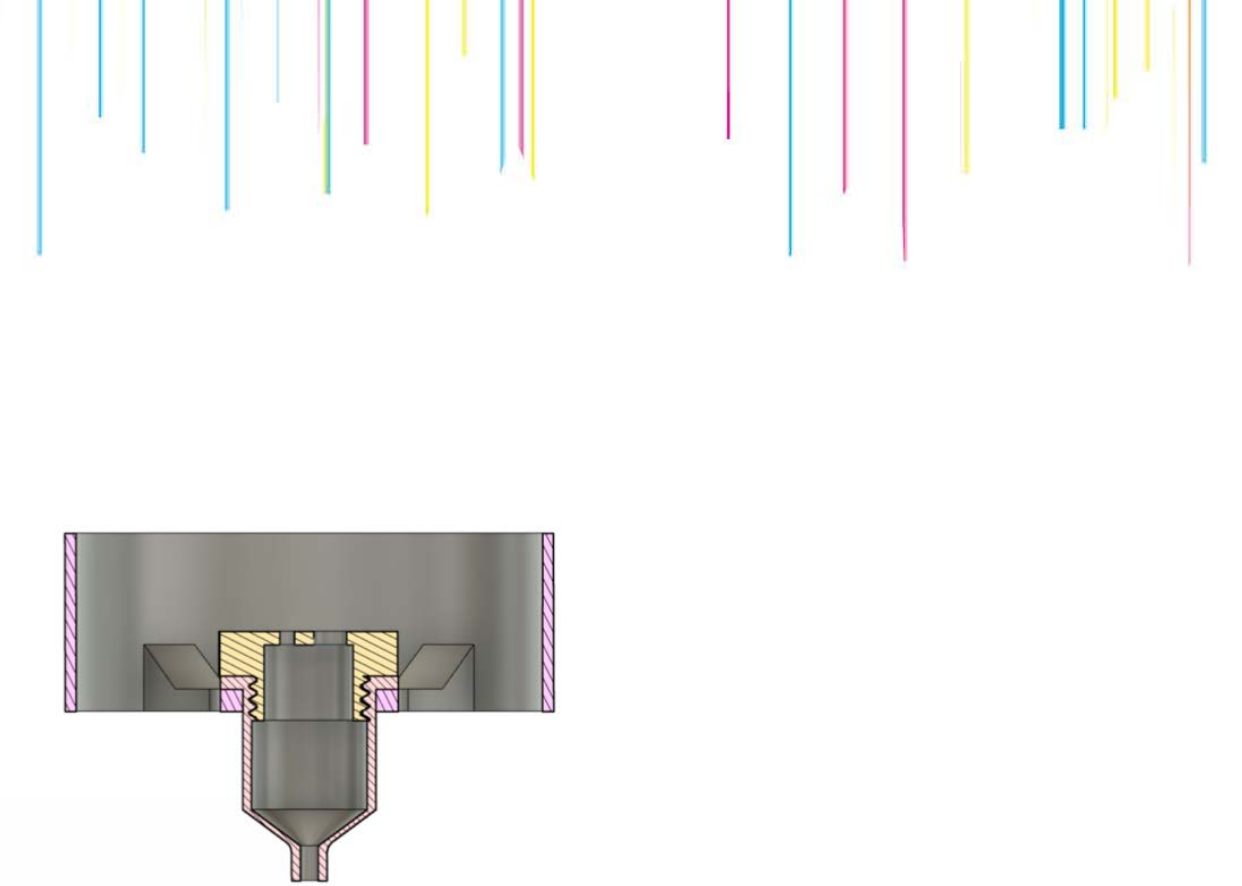

Figure 1: Section view of the moistening tool.

The customised weaving tool replaces the default milling spindle of a milling CNC machine, which will move around a predefined matrix with fixed anchor points, making a weaving movement and depositing the moistened fiber on the matrix.

A resin reserve tank was implemented with a pressure control system composed of a microcontroller, an HK1100C pressure sensor, a 300 psi air compressor, and an optocoupled relay (Figure 2). This adaptation allows manufacturing pieces with a large amount of fiber.

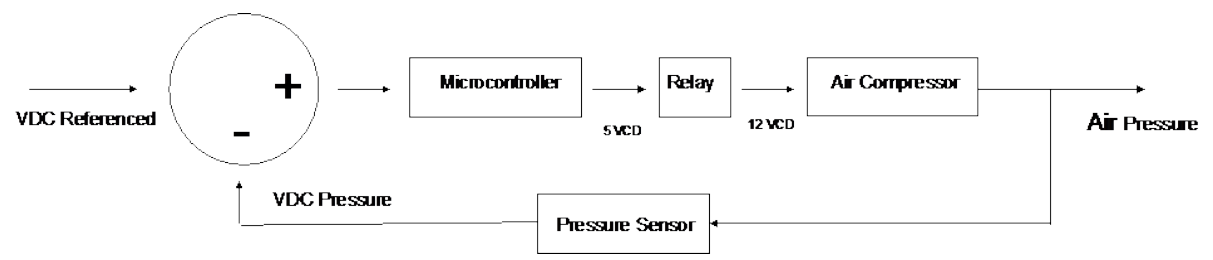

Figure 2: Tank Pressure Control System Diagram.

\subsection{Digital Prototypes}

Starting from a basic planar panel, the goal was to simulate the application of mechanical loads in different vectors, positions, and fixed vertices (Gil, 2019) (Figure 3). The combination of efforts was applied over three scenarios: first, a vertical load horizontally applied over the total extension of its superior edge; second a combination of lateral and vertical loads in the same geometric plane; and third considering only its weight with a fixed inferior edge (Table 2). 

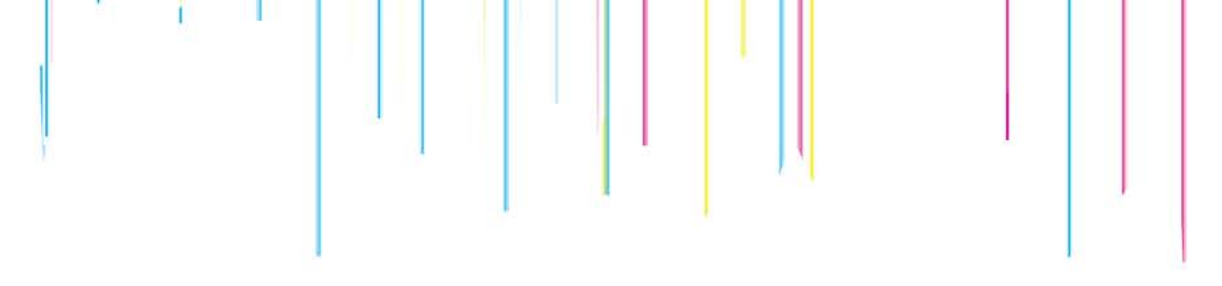

\begin{tabular}{llll}
$\begin{array}{c}\text { Axis I } \\
\text { Force type }\end{array}$ & $\mathbf{X}$ & $\mathbf{Y}$ & $\mathbf{Z}$ \\
\hline Compression & 0 & $10 \mathrm{~kg}$ & 0 \\
\hline Flexion & 0 & $10 \mathrm{~kg}$ & $11 \mathrm{~kg}$ \\
\hline Neutral & 0 & $10 \mathrm{~kg}$ & 0 \\
\hline
\end{tabular}

Table 2. Compression, flexion and natural applied forces corresponding to each scenario.

Firsts prototypes were done by randomly populating the inferior planar panel and superior edges with fixed points, which once each force was simulated, the edges acted as anchor points, limiting the vector movements through the panel. Setting the border conditions for the simulation. Using a topological optimization algorithm, both mechanical scenarios were tested in addition to a zero forces scenario, evaluating all the possible vectors as a result of the combination of the superior and inferior anchor points.
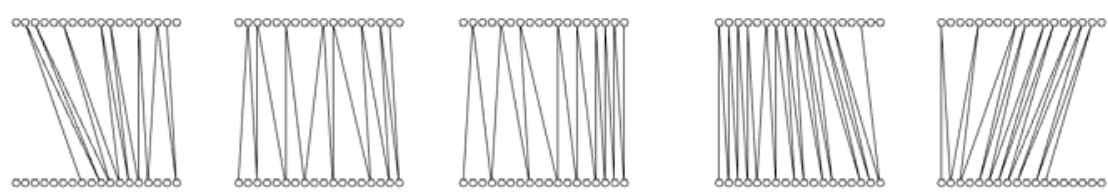

Figure 3. Random vector combinations from the 3.375 possibilities generated with the 15 by 15 points matrix diagram in a zero-force scenario.

Each vector represents a lineal trace of fiber, meaning that the zero-force scenario is a non-performative solution because it is equivalent to $100 \%$ of its mass and some fibers will not be under mechanical stress. The optimization goal then is the reduction of the number of vectors, leaving only those subjected to mechanical stress and those which contribute to the distribution of forces.

The selection of the performative vectors was developed by the discretization with points of the resulting graphical diagram, considering the lowest deflection value (Figure 4). In a second step, the outer edges and central axis points were selected, resulting in a clean matrix for the vector - and tool- to pass through. 


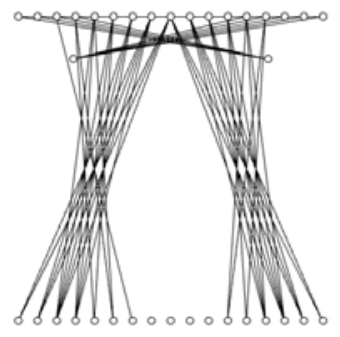

11576.777131

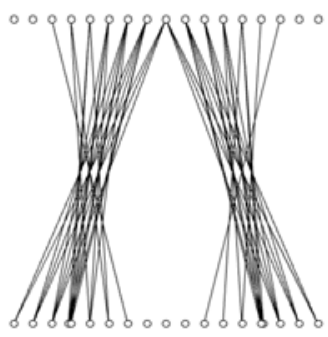

11576.777131

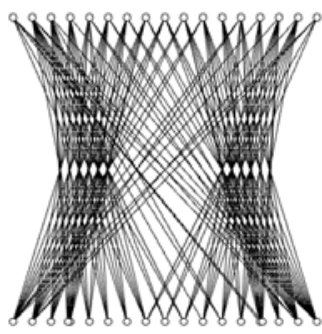

5788.372017

Figure 4: Points extracted from the optimization diagram of vertical forces applied over the horizontal superior edge with lowest vertices as anchor points. The values correspond to each deflection value.

In a 'finite element analysis software, the mechanical properties of the glass fiber were applied to the model, measuring the Von Mises Stress. The values were fed into the topology optimization model to have a distributed and balanced stress over the vectors reducing the displacement and elastic failure (Figure 5).
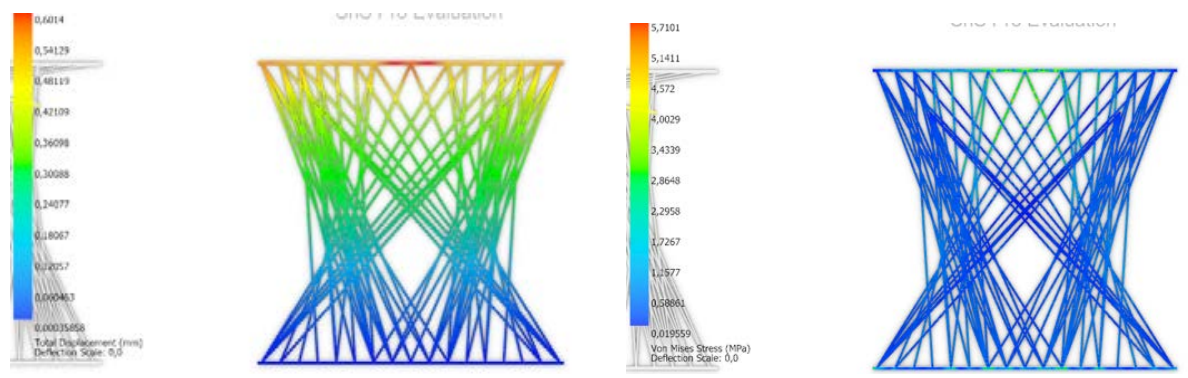

Figure 5. Displacement and Stress analysis diagrams.

Finally, each vector force was converted into polylines, which were sequentially organized and optimized by their length, resulting in a topological optimized array that will the path geometry for the glass fiber and tool. 

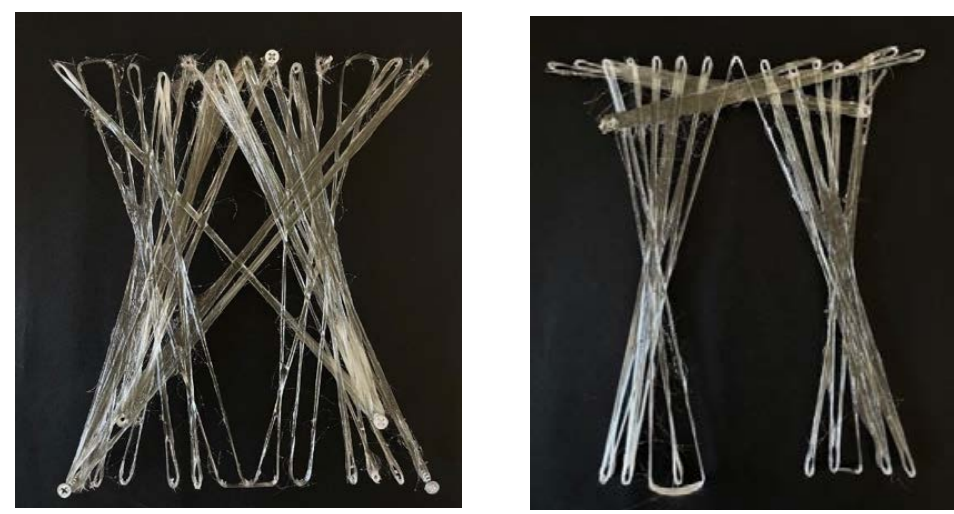

Figure 7. Final prototypes. In the left-hand pivots where manually controlled, in the right-hand pivots where adapted in the digital prototypes and toolpath.

This means that $100 \%$ of the used material is performing making the system smart in the sense that there is zero accumulation of non-active material. At the same time, the digital manufacturing process can produce flexible and fast different varieties of panels with a low investment of equipment.

\section{Further Steps}

In order to manufacture 1 to 1 scale prototypes, buckling studies and different fiber thicknesses should include in the simulation analysis. Also, measurements of the physical prototypes must be developed to compare them analytically with the digital prototypes

Acknowledgements. IPre intern students from the Pontifical Catholic University of Chile: Rodrigo Eterovic, Francisco Escalona and Augusto Galarce. FabHaus, Digital Fabrication Laboratory of the Faculty of Architecture, Design, and Urban Studies team: Antonia Valencia, Juan Pablo Valenzuela and Cristian Risco. Digital Futures PhD group from Tongji University.

\section{References}

AOC Resins. 2012. Guide to Fabricating FRP Composites. AOC World Headquarters. Ashland Inc. (2006). Derakane Consejos Para La Fabricación - Composite polymers. 


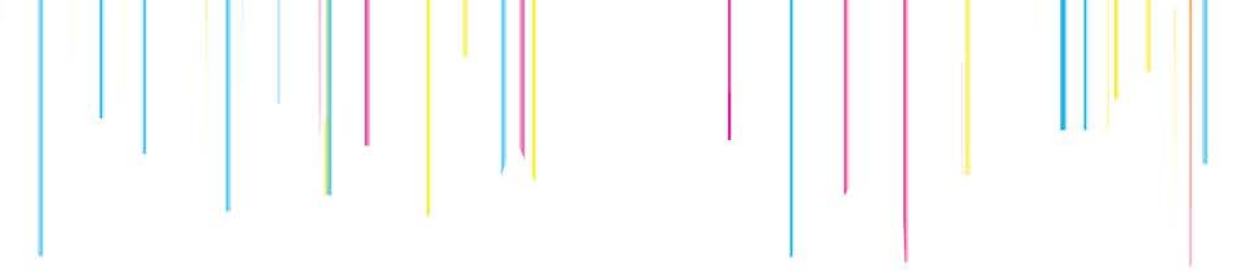

Burry, J., Sabin, J., Sheil, B., Skavara, M. (2020) Fabricate 2020 Making Resilient Architecture. UCL Press.

Dahy, H. (2019). Natural Fibre-Reinforced Polymer Composites (NFRP) Fabricated from Lignocellulosic Fibres for Future Sustainable Architectural Applications, Case Studies: Segmented-Shell Construction, Acoustic Panels, and Furniture. Sensors 2019, 19, 738.

Damborsio, N., Bodea, S., Zechmeister, C., Koslowki, V. (2019). Buga Fibre Pavilion: Towards an architectural application of novel fiber composite building systems. Ubiquity and Autonomy - 39th ACADIA Conference.

Dambrosio, N., Zechmeister, C., Bodea, S., Koslowski, V., Gil., Rongen, B., Knippers, J., Menges, A. (2020). Buga Fibre Pavilion: Towards an architectural application of novel fiber composite building systems. Fabricate 2020: Making Resilient Architecture p 142.

Dörstelmann, M., Knippers, J., Menges, A., Parascho, S., Prado, M., Schwinn, T. (2015). ICD/IT- KE Research Pavilion 2013-14: Modular Core- less Filament Winding Based on Beetle Elytra. Architectural Design, 85(5),p 54-5.

Gil, M; Rongen, B; Dabrosio, N; Menges, A. (2019). Structural optimization of coreless filament wound components connection system through orientation of anchor points in the winding frames. Proceedings of the IASS Annual Symposium 2019 Structural Membranes 2019.

Hensel, M; Menges, A. (2008). Inclusive Performance: Efficiency Versus Effectiveness Towards a Morpho-Ecological Approach for Design.

Hensel, M. (2012) Performative- Oriented Architecture: An integrated discourse and theoretical framework for architectural design and sustainability towards nondiscrete and non- anthropocentric architectures. PhD Thesis, University of. Reading.

Jaafar, J., Siregar, J. P., Salleh, S. M., Hamdan, M. H. M., Cionita, T., Rihayat, T. (2019). Important considerations in manufacturing of natural fiber composites: a review. International Journal of Precision Engineering and Manufacturing-Green Technology, 1- 18.

Knippers, J., Menges, A. (2014) "Fibrous Structures: An Integrative Approach to Design Computation, Simulation and Fa- brication for Lightweight, Glass and Carbon Fiber Composite Structures in Architecture based on Biomimetic Design Principles". Computer-Aided Design Journal.

Koustas, I., Papingiotis, T., Vosniakos, G., Dine, A. (2018). On the development of a filament winding robotic system. Procedia Manufacturing. Volume 17.

Narendra Kumar, Prashant K. Jain, Puneet Tandon, Pulak M. Pandey. (2019). Toolpath Generation for Additive Manufacturing Using CNC Milling Machine. Springer Singapore.

Vivanco, T., Valencia, A., Yuan, P. (2020). 4D printing: Computational Mechanical Design of Bi-dimensional 3D Printed Patterns over Tensioned Textiles for Lowenergy Three-dimensional Volumes. Proceedings of the 25th CAADRIA Conference. 